\title{
专访富碳纳米材料领域代表人物一一智林杰研究员
}

\author{
《物理化学学报》编辑部
}

北京大学化学与分子工程学院, 北京 100871

\section{Interview with the Pioneer in the Field of Carbon-Rich Nanomaterials: Professor Linjie Zhi}

\author{
Editorial Office of Acta Physico-Chimica Sinica \\ College of Chemistry and Molecular Engineering, Peking University, Beijing 100871, China. \\ Email:whxb@pku.edu.cn. \\ Published online: April 14, 2021.
}

富碳纳米材料是指具有高分子结构特征又具有碳纳米材料性能特征的新型纳米材料，不仅具有富碳高 分子明确的化学结构与组成, 而且其结构单元、分子骨架、孔道与表面结构、官能团种类与位置、杂原子 的引入与定位等重要的结构特征均可以通过结构单元的分子设计与可控的化学反应过程来实现较精确的 调节与控制。同时, 富碳纳米材料也具有碳纳米材料的重要性能特点, 如良好的导电性、高热稳定性、高 比表面积、高电化学活性等。该类材料不仅对材料学研究是一个全新的领域, 而且在能源、环境等领域的 实际应用中也具有非常重要的学术价值与经济价值。

我们有幸采访了富碳纳米材料专家, 《物理化学学报》 “石墨烯的功能与应用” 专刊的客座编辑智林 杰研究员, 为我们分享他在富碳纳米材料方面的研究经历以及富碳纳米材料在国内外的现状、应用、发展 方向等, 并对富碳纳米材料领域面临的一些重要问题进行了分析。

问题 1: 智老师好! 在您的科研生涯中从事过哪些 方面的研究? 您从什么时候开始从事富碳纳米材 料方面的研究?

回答: 我从1997年在中国科学院山西煤炭化学研 究所读博士研究生开始从事碳基(沥青)化学方面 的研究工作, 2000年到中国科学院化学研究所开 展博士后研究从事高分子化学方向的研究, 2003 年到德国马普协会高分子研究所从事博士后研究 工作, 开创性地将二者相结合, 正式开始了富碳纳 米材料方面的研究, 并于 2005 年开始担任马普高 分子所课题组组长, 之后一直致力于富碳纳米材 料的可控制备与应用领域的研究工作。

问题 2: 请介绍一下您的课题组在富碳纳米材料领 域取得的主要成果?

回答: 我的课题组长期从事富碳纳米材料的设计、
构建、功能调控及应用研究。1)提出以结构精确的 小分子或大分子为基本单元, 通过调控单元的化 学结构与交联反应在分子尺度上构建结构可控功 能可调的富碳纳米材料的研究策略; 2)通过热化 学途径实现富碳共轭网络结构的石墨烯化及化学 功能化, 以二维富碳高分子结构为单元通过可控 组装制备具有三维有序结构的富碳高分子纳米材 料, 设计和建构了具有独特化学杂化结构、骨架结 构、孔结构、复合界面结构的富碳纳米材料; 3)提 出了富碳结构与复合材料的维度匹配性概念, 开 辟了一条新的途径用以构建具有良好维度匹配性 的全一维、二维富碳复合电极材料, 为厘清化学掺 杂、孔道结构、共轭网络等结构信息在电化学存储 与转化应用 (超级电容器、锂离子电池、电催化等) 中的作用机制提供了一条新的思路; 4)发展了富 碳纳米材料的连续化批量制备工艺, 建立了基于 富碳纳米材料在透明导电薄膜、电极材料等应用 
中的评价工艺。

问题3: 请您简单回顾一下富碳纳米材料领域最近 几年的里程碑进展?

回答: 近年来, 有两类材料可以看作是富碳纳米材 料研究的领域里程碑式材料, 一类是以石墨烯为 代表的碳基纳米材料, 包括石墨烯、碳纳米管及富 勒烯等, 这类材料为富碳纳米材料的研究与发展 提供了理想的结构平台, 使得富碳纳米材料在这 些材料平台上通过自上而下的思路得以向深度和 广度上快速衍生, 大大促进了富碳纳米材料的研 究。因此, 从材料结构与功能来讲, 很多的功能化 石墨烯便属于富碳纳米材料的范畴。另外一类是 以有机共价框架结构为代表的网状结构材料, 这 类材料为富碳纳米材料自下而上的延伸与发展提 供了良好的材料结构基础, 进一步快速推动了富 碳纳米材料的研究与发展。

问题 4: 目前我国富碳纳米材料研究在国际上处于 什么水平?

回答: 从整体研究水平来看, 我国富碳纳米材料研 究已经走在了世界的前列。近年来, 在国家大力扶 持下, 经过不解努力, 我国在富碳纳米材料领域已 经打造了一支高水平的研究队伍, 建设了高水平 的研究平台, 特别是在国家鼓励创新、支持年轻人 发展的政策方针指引下, 在富碳纳米材料研究领 域涌现出一批思想活跃、创新意识强的青年学者, 取得了一系列高水平的研究成果。无论是在发表 论文数量, 还是在申请专利数上, 我国在该领域都 已经位居世界第一。

问题5: 富碳纳米材料领域有迫切需要突破的关键 技术吗? 请分析富碳纳米材料领域面临的一些重 要问题。

回答: 对大多数富碳纳米材料来说, 迫切需要解决 的问题是材料的精确可控制备与规模化生产技 术。目前, 大多数的富碳纳米材料还无法做到在分 子原子尺度上的精确的结构控制, 这导致对此类 材料的研究, 特别是深层次的物理与化学性质研 究具有不确定性, 从而使得我们对材料结构性能 相关性研究处于模糊层面, 严重影响了人们对此 类材料的科学认知。与此相关, 发展更先进的原位 表征技术和微观分析技术是富碳纳米材料研究领 域另外一个难题。随着科学技术的不断进步, 各种 精细的分析表征手段也越来越完善。但是如何针
对材料, 特别是介于有机材料与无机材料之间的 富碳纳米材料的结构在原子、分子尺度上进行精 确分析与表征仍然面临许多困难, 有待更多的学 者加入, 一起探询更精确高效的分析与研究方法。 发展高效低成本的规模化可控制备技术是此类材 料在实际应用中面临的最大挑战。在实验室里, 通 过精心控制各种反应条件, 可制备出高性能的富 碳纳米材料, 但是制备规模很小, 多是克级甚至毫 克级。一旦规模化制备, 由于放大效应的存在, 得 到的材料往往性能大幅下降。因此, 富碳纳米材料 领域也需要高素质的工程技术人员的参与, 从而 有效推动此类材料的商业化应用。

问题 6: 富碳纳米材料在哪些方面获得了实际应 用?

回答: 在大规模实际应用方面具有代表性的富碳 纳米材料有功能化石墨烯、基于前驱体的纳米碳纤 维、纳米多孔碳等。功能化石墨烯特别是基于化学 改性的功能化石墨烯是一类典型的富碳纳米材料, 目前已经在功能涂料、导电添加剂、功能填料等领 域进入规模化应用阶段。纳米碳纤维是一种高强度 的材料, 已经在航空、航天、汽车、船体等需要高 强度轻质材料的领域配合传统碳纤维实现了商业 化应用。纳米多孔碳具有极高比表面积, 是很好的 吸附材料, 已经广泛地应用于室内环境净化、污染 物处理和净化水体等领域。

问题7: 您可否预测和分析一下富碳纳米材料的现 状、发展方向?

回答: 目前, 富碳纳米材料无论在基础研究, 还是 在实际应用方面都取得了显著的进步, 材料种类繁 多、性质丰富多样、应用领域广泛, 并且发展潜力 巨大, 呈现出一片欣欣向荣的景象。然而, 从富碳 纳米材料的科学研究角度来讲, 目前的大多数研究 仍然处于早期阶段。富碳纳米材料是碳元素组成的 具有微观精巧结构的材料。富碳纳米材料未来的发 展需立足于它们的微观结构: 认识这些结构, 精确 设计与构建这些结构, 理解这些结构独特的性质, 并应用这些结构带来的独有性能。首先, 需要进一 步发展微观和原位表征技术, 特别是固态富碳材料 的原子尺度分析与表征方法, 充分认识富碳纳米材 料的结构特征, 揭示由结构带来的独特性质; 其次, 需要探索开发富碳纳米材料的独有应用, 而不是简 单的替换其它材料。同时, 继续开发大规模制备技 术, 使得富碳纳米材料能够大量精确制取, 而不是 
停留在实验内少量的制备; 再者, 扩大富碳纳米材 料实际应用范围, 受得 “杀手级” 应用, 使得富碳 纳米材料真正成为不可或缺的材料。

问题 8: 您对富碳纳米材料的学科发展有哪些期 望?

回答: 富碳纳米材料研究是国际前沿研究领域, 是 典型的化学、物理、材料等多学科深度交叉研究领 域。尽管在我国有越来越多的学者已经意识到这 一领域的重要性, 并开展了富有成效的研究与探 索, 但是也应该看到我们还存在很多不足。例如大 多数标志性的富碳纳米材料都是由外国人发现或 制备的; 重大的理论突破以及实验发现也大多来自 国外团队; 高性能富碳纳米材料关键制备技术也多 由国外团队首先提出。因此, 我国富碳纳米材料研 究应该立足于化学、物理、材料等我国优势学科的 同时, 通过多学科的深度交叉与融合, 充分挖掘材 料的结构特点和独有性质, 明晰材料结构和其性 能间的关系, 夯实此领域的理论基础, 就有机会做 出开创性的研究工作。特别是这类材料的精确结构
分析与结构精细化控制非常困难, 需要更多的青 年学者能够静心科研, 直面困难, 敢于挑战, 不能 急功近利, 不能追求 “短平快”, 做浅尝辄止的工作。 相信通过大家的共同努力, 在不久的将来就会在 这一领域出现越来越多的来自中国的原创性研究 工作。

问题9: 您对准备从事富碳纳米材料研究的青年学 者有何建议?

回答: 富碳纳米材料研究, 困难大, 挑战多, 但是 机会也多。建议青年学者: 1) 建立明确的研究方向, 深入地了解富碳纳米材料领域的国际前沿, 掌握其 面临的难点和挑战性问题, 选定自己感兴趣的研究 方向, 开展持之以恒的科研工作, 不要盲从热点, 进行恶性竞争; 2)加强学术交叉合作研究, 开展优 势互补、合作共享的研究模式, 注重多学科深入交 流与讨论；3)瞄准国家重大需求，重视基础研究的 同时也重视技术研究与工程研究, 使自己的研究成 果能够服务于国家经济与社会发展。

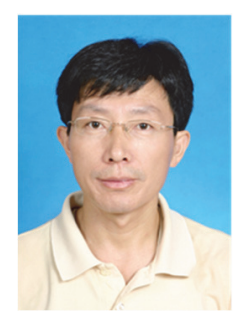

智林杰, 国家纳米科学中心研究员, 博士生导师。2000年在中国科学院山西煤炭化学研究所获化学工 艺学博士学位, 2000年至2002年在中国科学院化学研究所从事博士后研究。2003年至2007年在德国马 普协会高分子研究所(Max-Planck-Institute for Polymer Research)工作。从2005年开始任马普高分子所课 题组长(Project leader), 领导一个课题组从事富碳化学与能源材料方面的研究工作。2007年入选中国科 学院 “百人计划”加入国家纳米科学中心, 2014年获得国家杰出青年基金支持。主要从事基于富碳纳米 材料的结构调控、功能化与多元复合研究, 重点关注这些材料作为电极材料在储能器件与光电器件中 的应用, 作为催化剂材料在电化学催化与非均相催化中的应用, 以及材料在实际应用中的结构与功能 优化研究。截止目前, 在Nat. Commun. Angew.Chem.Int.Ed、、J.Am.Chem.Soc、Adv.Mater、Nano Lett、、Energy Environ. $S c i 、 A C S N a n o$ 等化学、材料、能源领域国际一流的学术刊物上发表学术论文 230 余篇。研究工作他人引用 25000 余次, 入选科㝐唯安 “高被引科学家”、爱思唯尔材料科学 “中国高被引学者”。研究成果多次被国际著名新闻媒体如Materials Views、Chemistry Views、Nanowerk、Newscientist等专题报道。先后承担国家863计划、科技部国际合作计划、科技部纳 米专项课题、国家自然科学基金项目等。目前担任中国颗粒学会能源颗粒委员会、中国地质学会纳米地质委员会、中国 能源学会、中国石墨烯产业技术创新战略联盟等理事, Scientific Reports、CrysEngComm、Advanced Materials Technology、 Science China Materials、New Carbon Materials等期刊编委。 